\title{
Successful treatment with cyclosporine and etanercept for deficiency of adenosine deaminase 2
}

Dai Keino ${ }^{1}$, Kensuke Kondoh ${ }^{1}$, Yuhwa Kim ${ }^{1}$, Akina Sudo ${ }^{1}$, Ryo Ohyama $^{1}$, Mizuho Morimoto $^{1}$, Hiroshi Nihira ${ }^{2}$, Kazushi Izawa ${ }^{2}$, Sachiko Iwaki-Egawa ${ }^{3}$, Tetsuya Mori ${ }^{1}$, and Akitoshi Kinoshita ${ }^{1}$

${ }^{1}$ St.marianna University School of Medicine Hospital

${ }^{2}$ Kyoto University

${ }^{3}$ Hokkaido Pharmaceutical University School of Pharmacy

May 7, 2020

\begin{abstract}
NA

ETHICS APPROVAL AND CONSENT TO PARTICIPATE

The study design was approved by the appropriate ethics review board. Informed consent was obtained from the patient and parents to participate.
\end{abstract}

CONSENT FOR PUBLICATION

Consent for publication was obtained from the patient and parents.

CONFLICT OF INTEREST

The authors have no conflicts of interest relevant to this article to disclose.

AUTHOR CONTRIBUTIONS

Dai Keino : Conceptualization (lead); Writing - original draft (lead); Writing - review and editing (equal). Kensuke Kondoh : Writing - review and editing (equal). Yuhwa Kim : Writing - review and editing (equal). Akina Sudo : Writing - review and editing (equal). Ryo Ohyama : Writing - review and editing (equal). Mizuho Morimoto : Writing - review and editing (equal). Hiroshi Nihira : Investigation (equal). Kazushi Izawa : Investigation (equal). Sachiko Iwaki-Egawa : Investigation (equal). Tetsuya Mori : Writing - original draft (supporting); Writing - review and editing (equal). Akitoshi Kinoshita : Conceptualization (supporting); Writing - review and editing (equal).

Keywords: Adenosine deaminase 2 deficiency, cyclosporine, anti-tumor necrosis factor, etanercept, pure red cell aplasia

To the Editor

Deficiency of adenosine deaminase 2 (DADA2) is an autoinflammatory disease caused by loss-of-function homozygous or compound heterozygous mutations in CECR1 (cat eye syndrome chromosome region 1). ${ }^{1,2}$ The phenotype of the DADA2 is widely heterogeneous with a variable age of onset. Manifestations include fever, early-onset lacunar stroke, livedo, portal hypertension, nodular vasculitis, immunodeficiency, 
hepatospenomegaly, pure red cell aplasia (PRCA), lymphopenia, cytopenia, and hypogammaglobulinemia. Treatment mainly consists of anti-tumor necrosis factor (TNF) agents. Steroids are also widely used for treatment and have shown variable success but often with flares of inflammation and vasculitis upon tapering. Cyclosporine (CyA), tacrolimus, cyclophosphamide, azathioprine, and methotrexate have all been used with little success. ${ }^{1,2}$ Although anti-TNF agents prevent strokes and improve the manifestations of vasculitis in DADA2, their efficacy for treating PRCA and bone marrow failure is less clear. ${ }^{3}$ Hematopoietic stem cell transplantation may be considered for patients with severe hematologic presentations. ${ }^{4}$

Here, we present here a young female patient who was initially diagnosed with idiopathic PRCA and later identified to have had compound a heterozygous mutation in ADA2 associated with DADA2. Administration of CyA and an anti-TNF agent (etanercept) successfully induced and maintained remission.

A 13-year-old girl was admitted to our hospital with fatigability and pallor. She has past histories of rash on her extremities at 8 months old and erythema multiforme at 11 years old. Her physical examination revealed palpebral conjunctival pallor and livedo reticularis of the lower limbs. Laboratory examinations indicated anemia (white blood cell count, $2900 / \mu \mathrm{L}$; neutrophils, $70.0 \%$; lymphocyte, $21.5 \%$; blasts, $0.0 \%$; hemoglobin $(\mathrm{Hb}), 4.6 \mathrm{~g} / \mathrm{dL}$; mean corpuscular volume, $81 \mathrm{fL}$; HbF, 0.4\%; Reticuro, $1.9 \times 10^{4} / \mu \mathrm{L}$; and platelet count, $\left.25.8 \times 10^{4} / \mu \mathrm{L}\right)$. Transaminase and lactate dehydrogenase were within normal ranges. IgA, IgM, and IgG were 42,46 , and $848 \mathrm{mg} / \mathrm{dL}$, respectively. Erythropoietin was 4,650 $\mathrm{mIU} / \mathrm{mL}$. Parvovirus, Epstein-Barr virus, and cytomegalovirus were serologically negative.

Bone marrow aspirate and biopsy indicated marked erythroid hypoplasia, and her nucleated cell count was $10.8 \times 10^{4} / \mu \mathrm{L}$. Chromosomal banding of bone marrow cells was 46,XX[14/14]. Bone marrow examination indicated no $\mathrm{T}$ cell receptor rearrangement. Computed tomography of the chest revealed no thymoma. Mutations affecting genes encoding ribosomal proteins causing Diamond Blackfan anemia were not detected.

She was diagnosed with idiopathic pure red cell aplasia (PRCA). Cyclosporine A (CyA) $4.5 \mathrm{mg} / \mathrm{kg} / \mathrm{day}$ was started at approximately 1.5 months after admission because she was dependent on red blood cell transfusion. Biopsy of the skin of the lower limb revealed necrotizing vasculitis, and a diagnosis of cutaneous polyarteritis nodosa (cPAN) was made (Fig 1). She responded to treatment with CyA and became transfusionindependent. The dose of CyA was gradually reduced, and approximately 10 months after diagnosis, she self-interrupted treatment with CyA. Six months after self-interruption, she became transfusion-dependent again. Resumption of CyA improved her anemia.

At the age of 17 years, no ADA2 enzyme activity was detected in the patient's plasma $(0.0 \mathrm{nmole} / \mathrm{min} / \mathrm{mL}$, normal range $2.38 \pm 0.95 \mathrm{nmole} / \mathrm{min} / \mathrm{mL}$ ). The results of CECR1 sequencing revealed compound heterozygous mutations: c.744delG p.Arg248fs and c.278T $>$ C p.lle93Thr. Thus, the patient was diagnosed with DADA2. Both parents and her sister were not carriers. Magnetic resonance imaging of the head showed no abnormal findings.

She continued to administer CyA but cPAN did not improve. She started administration of an anti-TNF agent (etanercept) in combination with $\mathrm{CyA}$ and noted improvement in her symptom sof cPAN such as livedo, edema, and fatigue in the lower limbs. More than a year has passed since starting etanercept with no adverse effects. Because $\mathrm{Hb}$ has remained at $11-12 \mathrm{~g} / \mathrm{dL}$, the amount of $\mathrm{CyA}$ has been tapered and is currently $0.5 \mathrm{mg} / \mathrm{kg} /$ day.

PRCA is a rare hematological disease characterized by bone marrow erythroid aplasia. PRCA may be congenital or acquired; the acquired form of chronic PRCA may present as a primary hematological disease in the absence of any other diseases or secondary to thymoma, lymphoproliferative disorders, infections, and collagen vascular diseases or after exposure to various drugs or chemicals. PRCA associated with DADA2 was described initially in three patients by Hashem et al. and Ben-Ami et al. and further confirmed in additional reports. ${ }^{5,6}$ A very early age of onset has been reported for PRCA (median, 0.3 years; range, 0.1-12 years). ${ }^{7}$

Initial treatment for PRCA includes the cessation of potentially deleterious drugs and careful observation for one month while making efforts to identify the cause of PRCA. Sawada et al. reported that CyA 
and corticosteroids induced remission in $74 \%$ and $60 \%$ of patients, respectively, and that discontinuance of maintenance therapy was strongly correlated with relapse. ${ }^{8}$ Although PRCA and other types of hematologic dysfunction associated with DADA2 generally do not respond well to immunosuppressive agents such as azathioprine and $\mathrm{CyA},{ }^{9}$ our patient achieved a response to $\mathrm{CyA}$. CyA may be useful for treating PRCA associated with DADA2.

PAN is considered a disease of adulthood with a median age of disease onset of approximately the 4th to 5 th decade of life. The annual incidence of childhood vasculitis is estimated to be 20 per 100,000 individuals under 17 years of age. ${ }^{10}$ Many children with PAN were found to have biallelic mutations in ADA2 ..$^{1,2,9}$ The mainstay of treatment for vasculitis/vasculopathy consists of anti-TNF agents (etanercept, infliximab, adalimumab). Although CyA was ineffective for cPAN in our patient, anti-TNF agents (etanercept) was effective, and at the age of 20 years, our patient has no neurological complications such as ischemic strokes, intracranial hemorrhage, and a wide range of neuropathies.

\section{REFERENCES}

1. Zhou Q, Yang D, Ombrello AK, et al. Early-onset stroke and vasculopathy associated with mutations in ADA2. $N$ Engl J Med . 2014;370:911-20.

2. Navon Elkan P, Pierce SB, Segel R, et al. Mutant adenosine deaminase 2 in a polyarteritis nodosa vasculopathy. N Engl J Med . 2014;370:921-31.

3. Ombrello AK, Qin J, Hoffmann PM, et al. Treatment strategies for deficiency of adenosine deaminase 2. N Engl J Med . 2019;380:1582-4.

4. Hashem H, Kumar AR, Muller I, et al. Hematopoietic stem cell transplantation rescues the hematological, immunological, and vascular phenotype in DADA2. Blood . 2017;130:2682-8.

5. Ben-Ami T, Revel-Vilk S, Brooks R, et al. Extending the clinical phenotype of adenosine deaminase 2 deficiency. J Pediatr. 2016;177:316-20.

6. Hashem H, Egler R, Dalal J. Refractory pure red cell aplasia manifesting as deficiency of adenosine deaminase 2. J Pediatr Hematol Oncol . 2017;39:e293-6.

7. Lee PY, Kellner ES, Huang Y, et al. Genotype and functional correlates of disease phenotype in deficiency of adenosine deaminase 2 (DADA2). J Allergy Clin Immunol 2020; doi: 10.1016/j.jaci.2019.12.908.

8. Sawada K, Hirokawa M, Fujishima N, et al. PRCA Collaborative Study Group: Long-term relapse-free survival and overall survival of patients with acquired primary idiopathic PRCA receiving cyclosporine A: A nationwide cohort study in Japan for the PRCA Collaborative Study Group.Haematologica . 2007;92:1021-8.

9. Hashem H, Kelly SJ, Ganson NJ, et al. Deficiency of Adenosine Desminase 2 (DADA2), an Inherited Cause of Polyarteritis Nodosa and a Mimic of Other Systemic Rheumatologic Disorders. Curr Rheumatol Rep . 2017;19:70.

10. Gardner-Medwin JM, Dolezalova P, Cummins C, et al. Incidence of Henoch-Schonlein purpura, Kawasaki disease, and rare vasculitides in children of different ethnic origins. Lancet . 2002;360:1197-202.

Figure legend

FIGURE 1. Skin of lower limb biopsy

Livedo reticularis was observed in both lower limbs to the acrotarsiums.

B, C. Necrotizing vasculitis (fibrinoid necrosis) accompanied by neutrophil infiltration was found in the subcutaneous fatty tissue. 


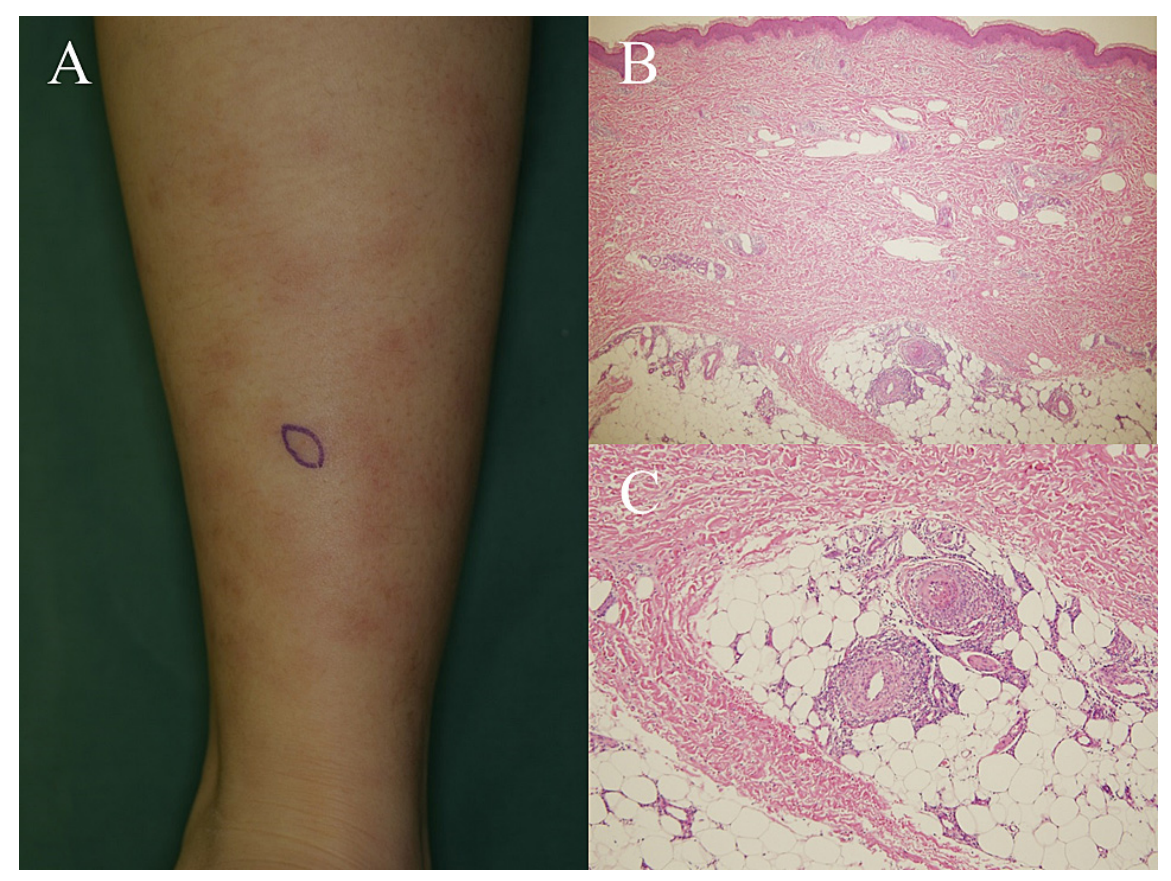

